\title{
Cultivation of Spirulina using Low-Cost Organic Medium and Preparation of Phycocyanin Based Ice Creams
}

\author{
A. Sukanya*, R. Meena and A. David Ravindran \\ Department of Biology, The Gandhigram Rural Institute-Deemed to be University, \\ Gandhigram- Dindigul, India \\ *Corresponding author
}

\section{Ke y w ords \\ Spirulina platensis, Zarrouk media, oil cakes, organic media phycocyanin, ice creams.}

\section{Article Info}

Accepted: 05 January 2020 Available Online: 10 February 2020

A B S T R A C T

Spirulina platensis is a filamentous Cyanobacterium with a high content of protein and essential vitamins that are commercially produced for feed and food supplementation. The present study was carried out to utilize the fermented oil cake filtrate as organic nutrient supplementation for Spirulina platensis cultivation. Commonly, the cultivation of S.platensis is done with conventional Zarrouk media that requires a high quality of $\mathrm{NaHCO} 3$. To reduce the cost of the medium different concentration of baking soda, sea salt, along with fermented oil cake filtrate were used as media for the cultivation of Spirulina platensis. Since there is a greater demand for organically grown Spirulina this study was undertaken. The main objective of this work is to cultivate Arthrospira S. platensis in edible oil cake incorporated medium. Higher biomass and pigment production concentration were scaled up to form labeled photobioreactor to outdoor trough cultivation. The nutritional content of the organic media was supplemented with the oil cake filtrate. The oil cake filtrate having a rich amount of nitrogen and phosphates will enhance the growth of Spirulina. In the present study, the Spirulina growth performance was recorded in both traditional and organic media of these the latter recorded better results. The phycocyanin of food-grade purity was incorporated in ice cream from organically cultivated biomass with foodgrade and its sensory evaluations were done.

\section{Introduction}

Spirulina, a Bluegreen alga having high nutritive value, exists in freshwater, brackish water and seawater habitats. It also can grow in warm temperatures and tropical regions and it is reported to grow abundantly in alkaline water bodies and salty lakes. S.platensis has been used as food for many centuries by different populations and only discovered in recent years. The annual production of the algae is about 10,000 tons which makes it the largest microalgal cultivation industry in the world, (Richmond, 
et al., 2000). Spirulina is a filamentous cyanobacterium with a high content of protein, essential vitamins that are commercially produced as food and feed supplementation.

The main objective of the present work is to cultivate the S.platensis using edible oilcake filtrate incorporated organic medium. Compared to other microalgae the overall production of Spirulina has increased from 5000 to 15000 tons /per year.

The production of Spirulina increase occurs due to the rich protein (60-70\%) composed of essential amino acids, vitamins include $\mathrm{B}_{12}$, polyunsaturated fatty acids, pigments and phenolic compounds due to their rich nutrient content has been marketed as a food supplement (Thajuddin and Subramanian 2005). The S.platensis have promising bioactive compounds compound viz, phycobiliproteins, phenolic compounds, organic acids, beta carotene, polyunsaturated fatty acids, polysaccharides etc.,(Belay et al., 2002 and Ozdemir et al., 2004)

These bioactive compounds have potential bioactive properties such as reducing the blood cholesterol levels, preventing cancer, stimulating the immune system to reduce the nephrotoxicity of pharmaceuticals. Monchai Dejsungkranont et al., (2013) optimized the algal biomass production experiments statistically designed variables varied viz, light intensity, $\mathrm{pH}$, two different strains of Cyanobacteria, different concentrations of Zarrouk medium, rate of aeration mixed with $\mathrm{CO}_{2}$ and temperature were studied through the of Taguchi method.

Dinesh Kumar et al., (2015) reported that the organic additive used enhanced the growth of Spirulina platensis. Centrifuge supernatant solution of Molasses was used as the organic additive for the Spirulina cultivation.
Molasses was used after centrifugation at $5000 \mathrm{rpm}$ for 10 minutes. The supernatant solution was used in different concentrations for enhancing the growth of Spirulina platensis. Rajeswari and Deepika, (2017) formulated the organic media for Spirulina cultivation by using agro wastes like beetroot, grapes, rice bran, the root of Casuarina, etc..

\section{Materials and Methods}

\section{Sample collection and pre culturing}

Spirulina platensis culture MK343101 was obtained from the Department of Biology, Gandhigram Rural Institute-Deemed to be University, Dindigul. The starter inoculums and culture were maintained in the traditional Zarrouk medium. The present study emphasis was on the use of the oil cake filtrate medium, baking soda along with that sea salts for the formulation of organic media for the cultivation of Spirulina platensis.

Different concentration of baking soda viz., $20-100 \%$, sea salt and ten $\mathrm{ml}$ of the fermented oil cake filtrate has been used for the media formulation. Preliminary work was done to standardize ten $\mathrm{ml}$ of the oil cake filtrate was used for $1 \mathrm{~L}$ of organic media.

\section{Microscopic identification}

The procured cultures were identified using the morphological characters (spiral shape) of blue-green algae (Spirulina) identified through the light microscope (Hernando Cortez 1519).

\section{Cultivation of Spirulina}

Spirulina was cultured in oil cake filtrate mediums in 1 L Pyrex glass photobioreactor under a controlled condition and Zarrouk medium as control. The cell concentration was analyzed at OD $560 \mathrm{~nm}$ Leduy et al., 
(1997). The $\mathrm{pH}$ of the medium was measured by using a $\mathrm{pH}$ meter. Spirulina platensis grows at a $\mathrm{pH}$ range of 8.9 to $9.5 \pm 0.2$ as it is maintained at the alkaline point.

Then culture was allowed to grow for 10 days and the biomass was harvested by filtration method, washed with distilled water to remove impurities and neutralize the $\mathrm{pH}$.

\section{Estimation of protein content}

The protein content of the harvested Spirulina biomass obtained by different organic media protein content was analyzed by Lowry's method (Lowry et al.,1951).

\section{Estimation of Beta-carotene}

Beta carotene is one of the major bioactive compounds present in the Spirulina platensis. The extraction of the Beta carotene was done through the methodology outlined by a with a slight modification of (Mandelli et al., 2012)

\section{Estimation of Phycocyanin}

Algal biomass sample of $0.1 \mathrm{~g}$ was mixed with $10 \mathrm{ml}$ of citric acid buffer ( $\mathrm{pH} 6.8)$. This mixture was kept in a deep freezer at $-20^{\circ} \mathrm{C}$ for 24 hours and thawed for every 6 hours respectively. After thawing the mixture was centrifuged at $5000 \mathrm{rpm}$ for 20 minutes.

The resulted supernatant was blue in color it denotes the presence of Phycocyanin pigment present in the biomass. The supernatant was read under a UV visible Spectrophotometer at $620 \mathrm{~nm}$. The crude phycocyanin was calculated through the formula (Boussiba and Richmond. 1979).

$$
\% \mathrm{CPC}=\frac{\mathrm{A} 620 * 10 \mathrm{ml} * 100}{7.3 * \mathrm{mg} \text { sample }}
$$

Where 7.3 is the Extinction coefficient of $\mathrm{CPC}$ at $620 \mathrm{~nm}$.

\section{Purity analysis of phycocyanin}

The purity of the phycocyanin can be calculated through the following formula Purity =A620nm/A280nm (Bennett and Bogorad, 1973).

\section{Concentrated the crude phycocyanin}

Phycocyanin was concentrated through the rotary vacuum evaporator. The extracted crude phycocyanin was poured into the rotary vacuum chamber at for $40^{\circ} \mathrm{C}$ at 20 mbar with $5 \mathrm{rpm}$ in 20 minutes to concentrate it. Then the concentrated Phycocyanin was freezedried and used for further analysis.

\section{The purity of the concentrated phycocyanin}

The purity of the concentrate phycocyanin was analyzed through the UV-VIS spectroscopy at $620 \mathrm{~nm}$ and 280nm.(RitoPalomares et al., 2001) wherein A620 is the maximum absorbance of C-PC and A280 is the absorbance of total proteins. The purity ratio meets if above 0.9 indicates it to be a food-grade fit for the food preparation.

\section{FT-IR analysis of concentrated Phycocyanin}

Concentrated crude phycocyanin was used for the FT-IR analysis to detect the antimicrobial functional compound through the ATR method. The surface functional group of Phycocyanin extracted from Spirulina, were unraveled by FT/IR 4700typeA. The Phycocyanin sample directly poured onto the crystal. The whole crystal-covered and performing quantitative or qualitative analysis. Amin Seyed Yagoubi et al., (2017)reported the characterization and evaluation of physicochemical properties of phycocyanin loaded solid lipid nanoparticles and nanostructured lipid carriers. 


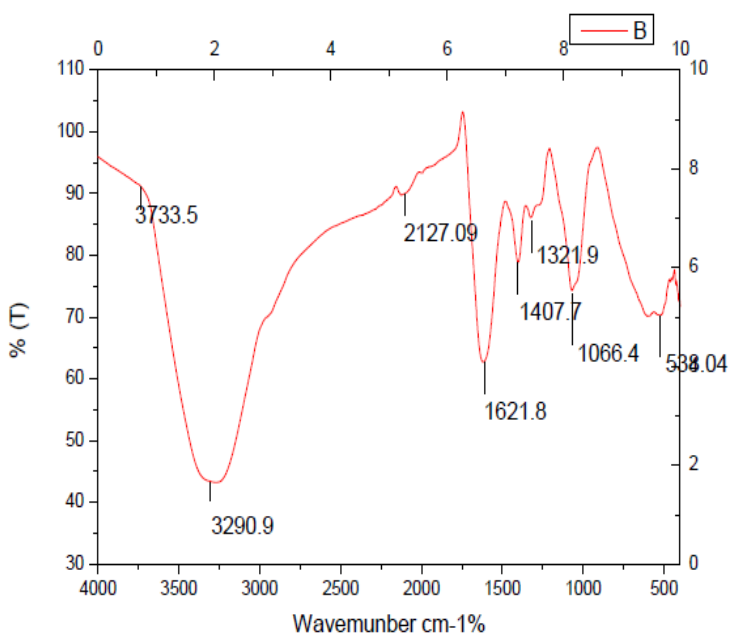

Figure.1 FT-IR Spectral graph of concentrated phycocyanin.

The IR spectral peaks indicate antimicrobial products of the phycocyanin bands at 1321 cm-1, $1407 \mathrm{~cm}-1$ (C-O-C), $1621 \mathrm{~cm}-1$ (CO of amide), $2127 \mathrm{~cm}-1(\mathrm{C}=\mathrm{C})$ and broadband $3290 \mathrm{~cm}-1$ (of $\mathrm{OH}$ and NH), Mostafa et al ,(2014) observed the antimicrobial substance from the S.platensis, in that study they found the spectroscopic analysis of the purified antimicrobial product IR spectrum showed bands at $1269 \mathrm{~cm}-1,1414 \mathrm{~cm}-1$ (C-O-C),
$1643 \mathrm{~cm}-1$ (CO of amide), $1563 \mathrm{~cm}-1(\mathrm{C}=\mathrm{C})$ and broadband $3441 \mathrm{~cm}-1$ (of $\mathrm{OH}$ and $\mathrm{NH}$ ).

\section{Microbial analysis of Phycocyanin}

The microbial content of the crude phycocyanin was analyzed standard plating method was followed. The standard plate count method was followed.One $\mathrm{ml}$ of concentrated phycocyanin was taken for the microbial analysis.

\section{Ice cream preparation}

Ice creams were prepared by the standard method, during the freezing time the concentrated phycocyanin added into $50 \mathrm{gm}$ of ice cream at $2 \mathrm{ml}, 5 \mathrm{ml}, 8 \mathrm{ml}, 10 \mathrm{ml}$.

Then mixed and frozen leave the ice cream in the freezer for a final freezer, until solid. The main sensory characteristics were evaluated as mentioned outlined by (Abd El Baky et al., 2015)

\section{Growth of Spirulina platensis in organic media as influenced by different concentration of sodium bicarbonate}

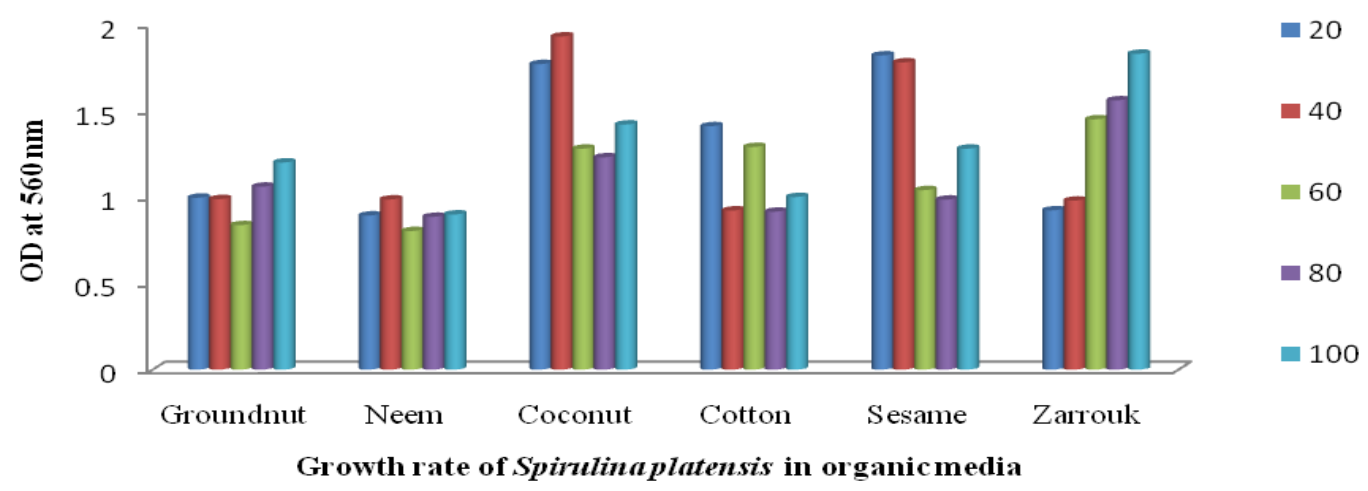

Figure.2 Growth of Spirulina platensis in organic media as influenced by different concentration of sodium bicarbonate 
After 10 days of culture, the absorbance of all-organic medium and control OD reached above 0.8 . The coconut filtrate with $40 \%$ sodium bicarbonate added medium reveals the highest growth OD as (1.93) and the lowest growth rate was observed in neem filtrate with $60 \%$ sodium bicarbonate medium growth OD (0.803).

Mahavir Joshi et al., 2014 demonstrated Zarrouk's media Supplemented along with rainwater, cows urine, well water cheese whey the maximum growth rate was observed at the fifteenth day of all media the maximum OD $(560 \mathrm{~nm})$ at 15 the day such as $1,76,1.53$, 1.75,1.44. (Fig .1) The biomass content of the all-organic medium along with control (Zarrouk) were as follows expressed as g/100 $\mathrm{ml}$ among the five organic medium as $20 \%$ groundnut oil cake filtrate medium showed $0.81 \pm 0.197 \mathrm{gm} / 100 \mathrm{ml}, 40 \%$ sesame medium shows the $1.14 \pm 0.32560 \%$ coconut medium $1.455 \pm 0.473$ and $80 \%$ groundnut medium $1.405 \pm 0.700$ and the $100 \%$ coconut oil cake medium $1.395 \pm 0.304$.

Jain and Singh (2013), they formulated a lowcost medium using cow dung ash for the biomass production they reported that Spirulina platensis is capable to grow in various kinds of culture media obtained 1.212 $\mathrm{g} / \mathrm{L}$ dry biomass.

Table.1 Optimization of biomass production as influence by different oil cake filtrate media with different concentrations of sodium bicarbonate

\begin{tabular}{|l|l|l|l|l|l|l|}
\hline S.no & $\begin{array}{l}\text { Substrates } \\
\text { oil cake }\end{array}$ & $\begin{array}{l}\mathbf{2 0 \%} \\
\mathbf{N a H C o 3} \\
(\mathbf{g m} / \mathbf{1 0 0 m})\end{array}$ & $\begin{array}{l}\mathbf{4 0 \%} \\
\mathbf{N a H C o} \\
\mathbf{( g m / 1 0 0 m l})\end{array}$ & $\begin{array}{l}\mathbf{6 0 \%} \\
\mathbf{N a H C o} \\
(\mathbf{g m} / \mathbf{1 0 0 m})\end{array}$ & $\begin{array}{l}\mathbf{8 0 \%} \\
\mathbf{N a H C o 3} \\
(\mathbf{g m} / \mathbf{1 0 0 m})\end{array}$ & $\begin{array}{l}\mathbf{1 0 0 \%} \\
\mathbf{N a H C o 3} \\
(\mathbf{g m} / \mathbf{1 0 0 m})\end{array}$ \\
\hline $\mathbf{1}$ & Neem & $0.7 \pm 0.197$ & $0.45 \pm 0.183$ & $0.71 \pm 0.339$ & $0.915 \pm 0.007$ & $1.185 \pm 0.714$ \\
\hline $\mathbf{2}$ & Coconut & $0.3 \pm 0.042$ & $0.49 \pm 0.113$ & $1.455 \pm 0.473$ & $1.09 \pm 0.395$ & $1.395 \pm 0.304$ \\
\hline $\mathbf{3}$ & Groundnut & $0.81 \pm 0.197$ & $1.05 \pm 0.197$ & $0.915 \pm 0.572$ & $1.405 \pm 0.700$ & $1.18 \pm 0.480$ \\
\hline $\mathbf{4}$ & Cotton & $0.23 \pm 0.02$ & $0.58 \pm 0.240$ & $0.975 \pm 0.26$ & $1.165 \pm 0.261$ & $1.16 \pm 0.353$ \\
\hline $\mathbf{5}$ & Sesame & $0.63 \pm 0.183$ & $1.14 \pm 0.325$ & $0.965 \pm 0.275$ & $1.235 \pm 0.148$ & $0.885 \pm 0.388$ \\
\hline $\mathbf{6}$ & Zarrouk & $0.43 \pm .0 .13$ & $0.67 \pm 0.14$ & $0.98 \pm 0.53$ & $1.45 \pm 0.10$ & $1.66 \pm 0.14$ \\
\hline
\end{tabular}

(gm/100 ml wet biomass)

Table.2 Determination of protein content in different oil cake filtrate media

\begin{tabular}{|l|l|l|l|l|l|l|}
\hline S.no & $\begin{array}{l}\text { Substrates(Oil } \\
\text { cake) }\end{array}$ & $\begin{array}{l}\mathbf{2 0 \%} \\
\text { NaHCo3 } \\
\text { g/100 gm }\end{array}$ & $\begin{array}{l}\mathbf{4 0 \%} \\
\text { NaHCo3 } \\
\text { g/100 gm }\end{array}$ & $\begin{array}{l}\mathbf{6 0 \%} \\
\text { NaHCo3 } \\
\text { g/100 gm }\end{array}$ & $\begin{array}{l}\mathbf{8 0 \%} \\
\text { NaHCo3 } \\
\text { g/100 gm }\end{array}$ & $\begin{array}{l}\mathbf{1 0 0 \%} \\
\text { NaHCo3 } \\
\text { g/100 gm }\end{array}$ \\
\hline $\mathbf{1}$ & Neem & $37.83 \pm 0.9$ & $56.31 \pm 0.75$ & $40.88 \pm 0.54$ & $37.16 \pm 0.54$ & $35.08 \pm 0.61$ \\
\hline $\mathbf{2}$ & Coconut & $40.23 \pm 0.52$ & $42.68 \pm 0.36$ & $35.11 \pm 0.09$ & $30.28 \pm 0.08$ & $29.39 \pm 0.61$ \\
\hline $\mathbf{3}$ & Groundnut & $33.43 \pm 0.79$ & $57.23 \pm 0.54$ & $42.68 \pm 0.54$ & $33.28 \pm 0.09$ & $30.35 \pm 1.05$ \\
\hline $\mathbf{4}$ & Cotton & $35.82 \pm 0.43$ & $53.23 \pm 0.46$ & $39.04 \pm 0.04$ & $41.6 \pm 0.26$ & $34.37 \pm 0.8$ \\
\hline $\mathbf{5}$ & Sesame & $42.15 \pm 0.69$ & $54.06 \pm 0.12$ & $40.06 \pm 0.59$ & $42.44 \pm 0.62$ & $32.64 \pm 0.66$ \\
\hline $\mathbf{6}$ & Zarrouk & $56 \pm 0.64$ & $61.39 \pm 0.54$ & $61.75 \pm 0.43$ & $60.67 \pm 0.54$ & $64.42 \pm 0.46$ \\
\hline
\end{tabular}


Aruna et al., 2008 reported $10 \mathrm{~g}$ of groundnut cake in $1 \mathrm{~L}, 15 \mathrm{~g}$ of neem cake, $15 \mathrm{~g}$ of press mud along with baking soda and one gram of sea salt used media enhance the growth of Spirulina and gave better results such as maximum growth rate were reported $1.0 \pm 0.1$ and chlorophyll $0.09 \pm 0.07 \mathrm{ug} / \mathrm{ml}$ and protein $45 \pm 3 \%$ and neem cake media $0.9 \pm 0.1$ chlorophyll $0.09 \pm 0.07 \mathrm{ug} / \mathrm{ml}$ and protein 45 $\pm 3 \%$.

Organic media from agro-waste like skin peel from beetroot(Beta vulgaris), leaves grape (Vitis vinifera), and extracts from rice bran and rice husk and root from Casuarina (Casuarina equisetifol) skin peel of beetroot grape leaves an extract of rice and improve the growth of Spirulina Rajeshwari and Deepika(2017). Table 1 provides the yield of biomass from the various concentration of sodium bicarbonate and oil cake filtrated treatment.

The table results revealed that the different concentrations of sodium bicarbonate along with the organic oil cake filtrate ass the media for the growth of Spirulina platensis for this study, it has to influence the growth of Spirulina at different levels.

Compared to the Zarrouk media lowest concentration of sodium bicarbonate and groundnut oil cake filtrate medium gave the highest biomass production with other media. Table 2 shows the protein content of the various concentration of sodium bicarbonate and oil cake filtrated treatment.

Table.3 Determination of Beta-carotene in different oil cake filtrate media

\begin{tabular}{|l|l|l|l|l|l|l|}
\hline S.no & $\begin{array}{l}\text { Substrates } \\
(\text { Oil cake) }\end{array}$ & $\begin{array}{l}\mathbf{2 0 \%} \\
(\boldsymbol{\mu g} / \mathbf{g m})\end{array}$ & $\begin{array}{l}\mathbf{4 0 \%} \\
(\boldsymbol{\mu g} / \mathbf{g m})\end{array}$ & $\begin{array}{l}\mathbf{6 0 \%} \\
(\boldsymbol{\mu g} / \mathbf{g m})\end{array}$ & $\begin{array}{l}\mathbf{8 0 \%} \\
(\boldsymbol{\mu g} / \mathbf{g m})\end{array}$ & $\begin{array}{l}\mathbf{1 0 0 \%} \\
(\boldsymbol{\mu g} / \mathbf{g m})\end{array}$ \\
\hline $\mathbf{1}$ & Neem & $1065 \pm 0.37$ & $2013 \pm 0.79$ & $1756 \pm 0.76$ & $1137 \pm 1.39$ & $541 \pm 0.59$ \\
\hline $\mathbf{2}$ & Coconut & $1038 \pm 1.88$ & $134 \pm 1.54$ & $1146 \pm 0.78$ & $584 \pm 0.979$ & $494 \pm 0.54$ \\
\hline $\mathbf{3}$ & Groundnut & $1054 \pm 1.81$ & $2184 \pm 0.65$ & $1877 \pm 2.26$ & $1141 \pm 1.39$ & $686 \pm 1.9$ \\
\hline $\mathbf{4}$ & Cotton & $1164 \pm 0.32$ & $2008 \pm 0.57$ & $1386 \pm 0.49$ & $698 \pm 0.99$ & $653 \pm 0.34$ \\
\hline $\mathbf{5}$ & Sesame & $1145 \pm 1.5$ & $1457 \pm 0.72$ & $1489 \pm 0.33$ & $998 \pm 0.65$ & $766 \pm 1.7$ \\
\hline $\mathbf{6}$ & Zaarrouk & $1040 \pm 0.59$ & $1421 \pm 0.32$ & $1488 \pm 0.45$ & $1546 \pm 0.74$ & $1817 \pm 0.34$ \\
\hline
\end{tabular}

It represents beta-carotene of the various concentration of sodium bicarbonate and oil cake filtrated treatment.

Table.4 Sensory evaluation of phycocyanin based ice cream

\begin{tabular}{|l|l|l|}
\hline Sensory characters & $\begin{array}{l}\text { Control without } \\
\text { phycocyanin }\end{array}$ & $\begin{array}{l}\text { Phycocyanin with different } \\
\text { concentration }\end{array}$ \\
\hline Colour & $9.7 \pm 1.02$ & $8.7 \pm 1.18$ \\
\hline Taste & $10 \pm 0.14$ & $9.4 \pm 1.23$ \\
\hline Texture & $10 \pm 0.43$ & $8.9 \pm 1.14$ \\
\hline Aroma & $9.9 \pm 0.02$ & $8.7 \pm 1.63$ \\
\hline Overall acceptability & $9.9 \pm 0.1$ & $8.7 \pm 1.20$ \\
\hline
\end{tabular}

(Mean \pm Standard deviation $\mathrm{n}=10$ )

Preparation of Ice-cream supplemented with concentrated phycocyanin

The Food Function Products (FFP)were prepared by using the standard ice cream preparation method. The food-grade pc purity (0.9) level of phycocyanin was incorporated into ice cream at different concentration levels (w/v) concentrated phycocyanin was 
added as an active ingredient at 2 to $10 \mathrm{ml}$ to the ice cream and commercial ice cream kept as a standard control.

A control ice cream without any phycocyanin was also prepared. All the ice cream were prepared in cold condition. After preparation stored in the freezing condition, ice creams were protected from light and temperature.

The ice creams were prepared with control ice cream The preparation with maximum phycocyanin incorporation $(10 \mathrm{ml} / 50 \mathrm{gm})$ in terms of color, texture, taste of the phycocyanin. These results indicate the positive effect of the phycocyanin.

These results revealed that ice cream prepared with higher phycocyanin concentration added ice cream is most likely to the panelist. And the taste leads to a good appreciation. These results revealed that phycocyanin based ice creams are the most favorite and very attractive when we prepared with the addition of phycocyanin.

The present study, investigated the comparative growth rate of Spirulina in Zarrouk's medium supplemented with different organic oil cake substrates. Spirulina cultivation was evaluated by lab-scale cultivation by using different organic based substrates as growth supplements.

Spirulina grows well in all the four substrates; the best growth, protein and pigment content were obtained in neem, groundnut and coconut filtrate supplemented medium. The experimental setup indicates that when Spirulina cultivated under varying concentrations of substrate supplementation, yield better growth and could be optimized as a low-cost media for the mass cultivation of Spirulina.

That organically cultivated product exhibits the functional food preparations. This present study extracts the phycocyanin from the organically cultivated Spirulina platensis, posses numerous bioactivities, has been used for the phycocyanin based ice cream preparation, the sensory evaluation of Food Function Products of ice creams are on the bar with the commercial ice creams.

\section{References}

Aruna S.J and David Ravindran.A,(2008) Cultivation of Spirulina sp using organic substrates, Journal of Applied and Pure Microbiology. Vol 2 (2) 483488.

Belay, A. (2002) The potential application of Spirulina (Arthrospira) as a nutritional and therapeutic supplement in health management. J.Am. Neutral. Assoc., 5, 27-48.

Bennet A. and Bogorad L. (1973). Complementary chromatic adaptation in filamentous blue-green algae. Journal of. Cell Biology. 58: 419-433.

Boussiba S. and Richmond A. (1979).Isolation and purification of phycocyanins from the blue-green alga Spirulina platensis. Arch. Microbial. 120: 155-159,

Dejsungkranont M, Phoopat N. et.al. Optimization of the Biomass Production of Arthrospira (Spirulina) Using the Taguchi Method. Journal of the Open Conference Proceedings 2012; 3: 70-81.

Dineshkumar. R, Umamageswari. P, Jayasingam $\mathrm{P}$ and Sampathkumar P.(2015) enhance the growth of Spirulina platensis using molasses as organic additives World Journal of Pharmaceutical Research vol..4 10571066.e 4, Is

Jain S. and Singh G.S., (2013). Low-cost medium formulation using cow dung ash for the cultivation of Cyanobacterium: Spirulina 
(Arthrospira) platensis, Journal of. Food Agriculture., 25 (9), 682-691.

Leduy.A, Therin.N(1997), An improved method for optical density measurement of the semimicroscopic blue algal Spirulina maxima Biotechnology and Bioengineering, 19 .1219-122.

Lowry,O.H., Rosebrough.N.J. Farr .A.L., and Randall R.J.,(1951) Protein measurement with the Foiln phenol reagent Journal of Biology Chemistry. 193 267-275.

Mahavir Joshi, Kiranjot Kaur, Tulika Mishra and Sanpreet Singh (2014) To evaluate Lab-scale Cultivation of Spirulina by using different substrates and to Evaluate its Chlorophyll and Protein content: International Research and Journal of Biological Science. Vol. 3(1), 22-30.

Mandelli F, Miranda V S, Rodrigues E, and Mercadante AZ, (2012). Identification of carotenoids with high antioxidant capacity produced by extremophile microorganisms. World Journal of Microbiology and Biotechnology 28:1781-1790.
Ozdemir, G.; Karabay, N.U.; Dalay, M.C, and Pazarbasi, B. (2004), Antibacterial activity of volatile component and various extracts of Spirulina platensis. Phytother Res., 18 (9), 754-757.

Rajeswari.P and Deepika.K, (2017) Formulation of organic media for the cultivation of Spirulina using agrowaste. International Research Journal of Biological Science vol (6)3,52-54 u

Richmond, A.(2000).Microalgal biotechnology at the turn of the millennium: A personal view. Journal of Applied. Phycology., 12: 441-451

Richmond,A(2000).Microalgal biotechnology at the turn of the millennium: A personal view. Journal of Applied Phycology., 12: 441-451.

Rito-Palomares M., Nunez L., Amador D.,(2001) Journal of. Chemistry. Technology. Biotechnology. 76 (2001) 1273.

Thajuddin, N., and Subramanian, G. 2005. Cyanobacterial biodiversity and potential applications in biotechnology. Curr. Sci. 89: 47-57.

\section{How to cite this article:}

Sukanya. A, R. Meena and David Ravindran. A. 2020. Cultivation of Spirulina using Low-Cost Organic Medium and Preparation of Phycocyanin Based Ice Creams. Int.J.Curr.Microbiol.App.Sci. 9(02): 392-399. doi: https://doi.org/10.20546/ijcmas.2020.902.049 